\title{
Mortality and incidence of cancer among sewage workers: a retrospective cohort study
}

\author{
Lennart Friis, Christer Edling, Lars Hagmar
}

\begin{abstract}
To study the incidence of and mortality from cancer among sewage workers a retrospective analysis was performed on a cohort of 656 men employed for at least one year at any one of 17 Swedish sewage plants during the years 1965-86. Assessment of exposure was done by classification of work tasks. Lower than expected total mortality (standardised mortality ratio $(S M R)=0.75,95 \%$ confidence interval (95\% CI) $0.58-0.97)$ and cardiovascular mortality (SMR = 0.61, 95\% CI 0.39-0.91) was found. This was interpreted as a result of the healthy worker effect. For all cancers combined the mortality (SMR $=1.08,95 \% \mathrm{CI}$ 0.68-1.67) and morbidity (SMR $=1.02,95 \%$ CI $0 \cdot 72-1 \cdot 38$ ) were comparable with those of the general population. There were increased incidences for brain tumours $(S M R=2 \cdot 19$, 95\% CI 0.45-6.39), gastric cancers (SMR = $2 \cdot 73,95 \% \mathrm{CI}, 1 \cdot 00-5 \cdot 94)$, and renal cancers $($ SMR $=1.68,95 \%$ CI $=0.35-4 \cdot 90)$. For lung cancer the risk was reduced $(S M R=0 \cdot 70,95 \%$ CI 0-15-2.05). Allowance for a latency period of 10 years from the start of exposure did not change the pattern. Logistic modelling was used to search for exposure-response relations. In a logistic model with the confounder age forced in, renal cancer had a significant positive relation with a weighted sum of employment times, where the weights describe the classification of exposure. No exposure-response relations were found for brain tumours or gastric cancers. The increased risks are based on small numbers of cases. A future follow up will add more conclusive power to the study. Specific exposures need to be identified to allow for a better dose-response analysis.
\end{abstract}

(British fournal of Industrial Medicine 1993;50:653-657)

Department of Occupational Medicine, University Hospital, Uppsala, Sweden

L Friis, C Edling

Department of Occupational and Environmental Medicine, University Hospital, Lund, Sweden L Hagmar
Occupational health care units responsible for sewage workers report several common acute symptoms-for example, transient episodes of gastrointestinal disturbances, irritation of the eyes and the upper respiratory tract, headache, and tiredness. Many of these short-term symptoms have been considered in earlier reports. Several studies and reviews of the effects of exposure to sewage focus mainly on infectious agents. ${ }^{1-3}$ Some symptoms can be explained by exposure to bacteria and bacterial toxins in waste water aerosols and dust from dried sludge. ${ }^{45}$ When we made a survey among 153 occupational health care units with responsibility for sewage plants there were, besides noise induced hearing loss, no long term effects reported from the health controls. In some places there has been concern, however, about cases of cancer of the stomach, the kidneys, or the lungs.

Among the many substances in the sludge there are carcinogens and mutagens ${ }^{6} 7$ and an increased frequency of urinary mutagens has been found in sewage workers, suggesting exposure to chemicals with mutagenic potential. ${ }^{8}$ In one case-referent study an increased risk for astrocytoma was found among people living near a municipal sewage plant. ${ }^{9}$ Recently a slight increase in mortality from cancer among sewage workers was found in a cohort study. The death rate from all cancers combined was greatest among the workers with the highest exposure. Significant excess mortality was noted for cancers of the larynx. Excess mortality, although not significant, was also noted for cancers of the stomach, the kidneys, and the lungs. ${ }^{10}$ Due to this increased concern about possible risk of cancer among sewage workers we were approached by the Swedish Water and Waste Water Works Association (VAV). To elicit whether sewage work may increase the risk of cancer, specifically in the brain, lungs, stomach, and the kidneys, a cohort of sewage workers was established and followed up. We present a retrospective analysis of mortality from and incidence of cancer in this cohort.

\section{Materials and methods}

SUBJECTS

Data from a cohort of sewage workers, men as well as women, were collected from the municipal 
sewage plants in 17 small and medium sized cities in the southern half of Sweden. In 1986 the population in these cities ranged from 33000 to 157 000. The inclusion criterion was employment for a minimum of one year at one of these plants during 1965-86. The method of identifying the workers varied much depending on the local circumstances. Some municipalities keep computerised records of all employees where their specific work sites can be found, whereas others have only rudimentary records that do not allow for a straightforward way of sure identification of former employees. On some of these plants the efforts to accomplish complete reports included asking older retired workers about old colleagues. Requested data for each person in the cohort were reported on a specific protocol that was filled in at each plant or at the municipal authority responsible for the sewage plant. The data contained the identifying data of the municipality and the worker, times of employment, information on main work tasks, and smoking habits.

\section{EXPOSURE}

The information on work tasks was the base for the exposure classification. An exposure subgrouping was made by the VAV and the union. The different subgroups were laboratory work or similar, work at the waste water processing plant, sludge pipe flushing, or exhaustion and work at sewage pump stations. The exposure was considered low in the laboratory work and high in the pumping stations where the workers have a substantial exposure to waste water aerosol. The rationale behind this classification was the degree of general exposure to waste water and aerosols and the general impression of impurity. Workers with more than one type of exposure during one employment were allocated to the group where they had most exposure, and in cases of doubt the highest exposure in question was chosen. Three employment periods with separate exposure classes were allowed for in the reported data. Three classes of smoking habits were defined-namely, smoker, non-smoker, and unknown. All present smokers and all who had finished smoking less than 10 years at the time of filling the protocols, were classified as smokers.

\section{ANALYSIS}

Information about mortality was collected from the National Death Registry of Statistics Sweden. The officially adopted underlying cause of death is noted by the International Classification of Diseases (ICD) code in this register. The information on incidence of cancer was collected from the Swedish Cancer Registry at the National Board of Health and Welfare.

Analyses were performed with the epidemiologi- cal computer program EPILUND at the Department of Occupational and Environmental Medicine, the University Hospital in Lund, Sweden. This program calculates a person-years of observation matrix, and expected values based on the cause, sex, and five year age group specific national death rates and incidences of cancer. Finally it also computes standardised mortality ratios (SMRs) and 95\% confidence intervals (95\% CIs) according to the Poisson distribution or to the $\chi^{2}$ distribution if the expected values are greater than 10. The national death rates and incidences of cancer included in the program package at the time of analysis were up to 1985 . We had data until 1987 , however, and to allow for an analysis of the study period $1965-87$ the national death rates and incidences of cancer for 1986 and 1987 were approximated to the data for 1985. All analyses were performed only on the men. Only ages up to 79 years were considered because of the more uncertain diagnostic procedures at older ages.

Exposure-response relations were analysed by logistic regression with the statistics package EPILOG on a personal computer (Epicenter Software, Pasadena, Ca, USA). As a first attempt at logistic modelling, the total time of exposure (=employment) was used as the exposure variable. To evaluate the influence of different work categories on the risk for cancer we defined an exposure index (EI), a weighted sum of the time of employment $(\mathrm{T})$, which we used as the exposure variable in a second series of regression analyses. The weights were set to $\mathrm{W}_{1}=0$ for laboratory work, $\mathrm{W}_{2}$ $=1 / 3$ for work at the plant, $W_{3}=2 / 3$ for sludge pipe flushing or exhaustion, and $\mathrm{W}_{4}=1$ for work at the pumping stations. When measurements of exposure are lacking this is one possible way of describing the assumed relations between the classes of exposure.

$$
\mathrm{EI}=\sum_{\mathrm{i}=1}^{4} \mathrm{~W}_{\mathrm{i}} \mathrm{T}_{\mathrm{i}} ; \mathrm{W}_{1}=0, \mathrm{~W}_{2}=1 / 3, \mathrm{~W}_{3}=2 / 3, \mathrm{~W}_{4}=1
$$

was calculated for each worker in the analysis. The latency requirement of at least 10 years from the first exposure until a diagnosis of cancer was applied for inclusion as a case in the analysis. For the cases only time until diagnosis was considered. Odds ratios (ORs) with $95 \%$ CIs were calculated from the logistic regression models.

\section{Results}

Among the reported 712 subjects was one man who had emigrated and one man whose national registration number was incomplete and impossible to track. Thus the loss to follow up was $0.3 \%$. This gave a cohort consisting of 710 persons of which 656 were men. For these men the average time of 
Table 1 Observed (O) and expected (E) numbers of deaths among Swedish male sewage workers during the study period 1965-87

\begin{tabular}{lllll}
\hline & Person-years $O$ & $E$ & $S M R(95 \% C I)$ \\
\hline $\begin{array}{l}\text { All causes of death } \\
\text { Cardiovascular death }\end{array}$ & 9534 & 62 & $82 \cdot 2$ & $0.75(0.58-0.97)$ \\
$\quad$ (ICD-8 390-458) & 24 & 39.6 & $0.61(0.39-0.91)$ \\
$\begin{array}{l}\text { All malignant tumours } \\
\text { (ICD-8 140-209) }\end{array}$ & 21 & 19.4 & $1.08(0.68-1.67)$ \\
\hline
\end{tabular}

employment at a sewage plant until the end of 1986 was 12.6 (SD 8.4) years. The longest employment was 49 years and the shortest was one year. The total time of work at a sewage plant for the male subcohort was 8258 years. The mean age of the male cohort members still alive at the end of 1987 was $49 \cdot 1$ (SD 14.1) years. We had information about smoking history for 502 men (76\%). Fifty per cent were smokers or had stopped smoking less than 10 years previously.

\section{MORTALITY}

During the study period $1965-87$ the 656 men contributed 9534 person-years of observation. Sixty two had died compared with 82.2 expected (SMR $=0.7595 \%$ CI $0.58-0.97$; table 1 ). This significantly decreased mortality was mainly due to a significant deficit in cardiovascular deaths (SMR = $0.61,95 \%$ CI $0.39-0.91$ ). No significant increase in mortality from malignant tumours was found.

\section{CANCER MORBIDITY}

An analysis of cancer morbidity at different cancer localisations (table 2) showed increased risks for tumours in the brain (including the meninges), the stomach, and the kidneys. No increased risk for all cancers combined, or for lung cancer (cancer of the trachea, bronchi, lungs, or pleura) were found. The estimates were calculated taking into account two latency periods, zero and 10 years, from start of exposure until contribution to the person-year of observation matrix. For all malignant tumours combined the analyses gave SMRs close to 1 with 95\% CIs embracing unity in a fairly symmetrical manner. There was a significantly increased risk for stomach cancer $(\mathrm{SMR}=2 \cdot 73,95 \%$ CI $1 \cdot 00-5 \cdot 94)$ in the analysis with no latency requirement. When the 10 years latency condition was applied in the person-year calculations the risk was still increased although not significant $(\mathrm{SMR}=2.49,95 \% \mathrm{CI}$ $0 \cdot 68-6 \cdot 38$ ). The increased SMRs for tumours of the brain in the zero and 10 year latency categories were 2.19 and 2.37 , and for the kidneys 1.68 and 2.39 respectively, with wide $95 \%$ CIs. For lung cancer there was a lower than expected incidence, although this was not significant.

In the logistic regressions age was modelled linearly in the logit. Age is a strong confounder of total time of employment and the exposure index. Therefore age was forced in the logistic regressions for these variables. For smoking there were no data for about a quarter of the cohort. Thus when performing regressions with smoking included as a variable the study group was reduced considerably by exclusion of those with no data about smoking. In most cases, when including the smoking variable in the analyses of the specific cancers, the regression computations did not converge.

For all cancers combined age was, as expected, associated with the incidence of cancer. For a 10 year increase in age the OR was 1.95 (95\% CI $1 \cdot 44-2 \cdot 63$ ). In the logistic model with age and smoking as regression variables the adjusted OR for smoking was $2 \cdot 10(95 \%$ CI $0 \cdot 78-5 \cdot 59)$. For all cancers combined the two logistic models for the exposure variables with age and smoking forced in showed no significant positive relations. For an increase of 10 years in total time of employment the adjusted OR was $1 \cdot 40$ (95\% CI $0 \cdot 82-2 \cdot 41)$. For an increase in exposure index of 10 units the adjusted OR was $1 \cdot 40$ (95\% CI 0.74-2.65).

Cancer of the stomach and lung cancer had no significant association with total time of employment or exposure index in the bivariable models

Table 2 Observed (O) and expected (E) numbers of malignancies among Swedish male sewage workers during the study period 1965-87

\begin{tabular}{|c|c|c|c|c|c|c|c|c|}
\hline & \multicolumn{4}{|c|}{ No latency period } & \multicolumn{4}{|c|}{10 year latency period } \\
\hline & $\begin{array}{l}\text { Person-years } \\
\text { of observation }\end{array}$ & $O$ & $E$ & $S M R(95 \% C I)$ & $\begin{array}{l}\text { Person-years } \\
\text { of observation }\end{array}$ & $O$ & $E$ & $\operatorname{SMR}(95 \% C I)$ \\
\hline $\begin{array}{l}\text { All malignant tumours } \\
\text { (ICD-7 140-209) }\end{array}$ & 9443 & 37 & $36 \cdot 1$ & $1 \cdot 02(0 \cdot 72-1 \cdot 38)$ & 4331 & 26 & $26 \cdot 3$ & $0.99(0.64-1.40)$ \\
\hline $\begin{array}{l}\text { Brain including meninges } \\
\text { (ICD-7 193.0) }\end{array}$ & & 3 & $1 \cdot 37$ & $2 \cdot 19(0.45-6 \cdot 39)$ & & 2 & $0 \cdot 85$ & $2 \cdot 37(0.29-8 \cdot 55)$ \\
\hline $\begin{array}{l}\text { Stomach } \\
\text { (ICD-7 151) }\end{array}$ & & 6 & $2 \cdot 20$ & $2 \cdot 73(1.00-5 \cdot 94)$ & & 4 & $1 \cdot 61$ & $2.49(0.68-6.38)$ \\
\hline $\begin{array}{l}\text { Kidneys } \\
\text { (ICD-180 }\end{array}$ & & 3 & $1 \cdot 79$ & $1.68(0.35-4.90)$ & & 3 & $1 \cdot 25$ & $2.39(0.49-6.99)$ \\
\hline $\begin{array}{l}\text { Lungs } \\
\text { (ICD-7 } 162 \cdot 0-162 \cdot 2)\end{array}$ & & 3 & $4 \cdot 27$ & $0 \cdot 70(0 \cdot 15-2 \cdot 05)$ & & 2 & $3 \cdot 16$ & $0.63(0.08-2.29)$ \\
\hline
\end{tabular}


with age. Logistic regression for smoking did not converge for either of these two cancers. We had data about smoking for two of the three lung cancer cases; both were smokers. Brain tumours had no significant association with age, smoking, total time of employment, or exposure index.

Cancer of the kidneys had no significant association with age or the total time of employment, and the analysis for smoking did not converge. For the exposure index there was a significant positive relation with $\mathrm{OR}=2.95$ for an increase in exposure index of 10 units $(95 \% \mathrm{CI} 1 \cdot 22-7 \cdot 12)$. The logistic model with the confounder age forced in gave a significant positive relation for exposure index with an adjusted OR of 2.60 for an increase in exposure index of 10 units (95\% CI 1.00-6.80).

\section{Discussion}

This study showed a significant reduction in total mortality among sewage workers when compared with the general population. A large part of this reduction was due to a significantly decreased risk for cardiovascular deaths. We found no significant increase in mortality or morbidity for all malignant tumours combined. For three of the four specific cancer sites towards which we originally directed our interest, the analysis showed higher than expected SMRs. These were tumours of the brain, stomach, and kidneys. By contrast, lung cancer showed a decreased risk.

The collection of data for retired personnel in some municipalities was done without reliable records. This will introduce a risk of recall bias, if a colleague with a severe disease is more likely to be remembered than someone without such a disease. This could lead to an overestimation of possible risks in a retrospective analysis. Our impression, however, is that those responsible for assembling the data have taken all reasonable efforts not to miss any of the workers qualified for the cohort. Furthermore, the plants in question are small enough such that everyone should have had personal knowledge of all the other workers. This leads us to believe that the missed persons are few, if any. The effect on SMR of a missed case would be a reduction, whereas by contrast one missed "healthy subject" would increase the SMR by reducing the expectation value. This effect is unsymmetrical depending on whether the missed subject has the studied disease or not. As the numbers of cases are few the relative effect on the SMR by one missed case would be greater than by one missed subject without the disease.

Small cohort studies require a pronounced excess mortality to produce statistically significant results, and may leave moderately increased risks undetected. The power of this study is estimated at the 0.05 level (one sided) by the method for cohort studies when comparing with an external standard, as presented by Breslow and Day. ${ }^{11}$ For all cancers combined, when taking into account the 10 year latency requirement, this study has $75 \%$ power to detect a true relative risk (RR) of $1 \cdot 15$. For $R R=$ 1.1 the power is $13 \%$. For the specific cancers the power of this study is low, as the expected numbers of cases are low. For renal cancer, for example, the power to detect an $R R$ of 2.0 is $16 \%$.

The validity of the lower than expected mortality is questionable due to the limited size of the cohort and the use of the general population as the reference. The general population includes groups with higher mortality than workers with long-term employment. In cohorts of active workers the observed number of deaths often falls short of expected numbers calculated from national averages. This is due to the healthy worker effect, which tends to reduce the RRs in cohorts of active workers. Because these sewage workers have a physically demanding job it is reasonable to assume a selection of relatively healthy persons into this cohort.

The lower than expected SMR for lung cancer may, at least in part, be due to the use of the national rates for computing the expected values. The 17 municipalities from which we collected our cohort did not include the three largest cities in Sweden. These three cities differ from the rest of Sweden in mortalities and morbidity rates. The incidences of lung cancer are substantially higher, whereas the differences for the other specific incidences of interest in this study are less pronounced.

Our finding of no significant increase in either mortality or morbidity for all malignant tumours combined agrees with the result of Lafleur and Vena. ${ }^{10}$ From theoretical considerations no increase is to be expected if an increase is anticipated only in a few specific cancers. This lumped diagnosis of all cancers combined leads to a diagnostic dilution and causes an underestimate of any possible increased risk. This study by Lafleur and Vena ${ }^{10}$ also showed increased SMRs for cancers of the stomach and kidneys. The numbers of cases were small, as in our study, however, and the results were not significant. For laryngeal cancer they presented a significantly increased risk based on three cases. In our cohort there are so far no cases of laryngeal cancers.

The only significant result we found was the increased SMR for cancer of the stomach in the analysis without the latency requirement. Applying the latency requirement led to a reduction of the expected numbers of cases. Despite this, the results point in the same direction whether the latency period is applied or not. When the 10 year latency period was applied the SMR for renal cancer increased from 1.68 to $2 \cdot 39$. This suggests the possibility of a causal relation between the 
exposures in waste water work and renal cancer. For the other cancers there is no such strengthening of our hypotheses. If the SMRs we have found point toward increased risks more conclusive results would be expected in a future prospective follow up.

Although the associations found are weak, we attempted to strengthen the evidence by establishing dose-response relations. Thus we made the logistic regression analyses, although the few cases is also a weak point here. The assessment of exposure we used is rather crude, and no hygienic measurements were available. The weight factors introduced for supposed levels of exposure only reflect a general impression of the environmental impurity in the waste water treatment process. Parallel to the flow in the sewage system many changes occur to the waste water and the sludge. Chemicals are added, spontaneous and induced biological and chemical processes take place, substances and matter are eliminated by chemical and mechanical means from the water, and the sludge is concentrated by reduction of the water content. Our assumption that the highest exposure occurs in the pumping stations at the beginning of the process could as well be completely reversed for some potentially harmful factors. These may not even be present in the water before it has come into the process in the plant, where chemicals are added. The microbial content of the waste water is certainly extremely variable in the different parts of the process. A connection between cancer of the stomach and bacteria has recently been described. Infection with Helicobacter pylori is associated with chronic atrophic gastritis, which is a precursor of gastric adenocarcinoma. Two nested case-control studies have shown an increased risk of gastric adenocarcinoma in subjects infected with Helicobacter pylori. ${ }^{12} 13$

This specific bacteria is widespread in the general population but has not been reported to be recovered from samples of sewage water. Acute gastrointestinal disturbances are common among sewage workers, but the prevalence of subclinical chronic gastritis in such workers is unknown.

The relation between renal cancer and the exposure index used is positive and significant, but not for the unweighted total exposure time. This may of course be a chance finding, but another possibility is that some agent in waste water acts as a renal carcinogen. The lack of relations for brain tumours and cancer of the stomach might be due to these tumours being caused by other factors that are poorly assessed by our exposure index.

\section{Conclusion}

Our study did not find an increase in total mortality from or incidence of cancer among sewage workers. There were non-significant excess risks for certain cancer sites-namely, brain, kidneys, and stomach, with a dose-response relation for cancer of the kidneys. To be able to analyse the risks of specific cancers in more detail there is a need to make a detailed survey of the exposures in sewage work. A future follow up of this cohort will increase the person-years of observation which, together with better exposure data, will allow for more conclusive results.

Requests for reprints to: $\mathrm{Dr}$ Lennart Friis, Department of Occupational Medicine, University Hospital, S-751 85 Uppsala, Sweden.

1 Clark CS, Cleary EJ, Schiff GM, Linnemann CC, Phair JP, Briggs TM. Disease risks of occupational exposure to sewage. Fournal of the Environmental Engineering Division (American Society of Civil Engineers) 1976;102:375-88.

2 Skinhøj P, Hovind-Hougen $\mathrm{K}$, Hollinger FB, Lous P. Infectious liver diseases in three groups of Copenhagen workers: correlation of Hepatitis A infection to sewage exposure. Arch Environ Health 1980;36:139-43.

3 McCunney RJ. Health effects of work at waste water treatment plants: a review of the literature with guidelines for medical surveillance. Am $\mathcal{F}$ Ind Med 1986;9:271-9.

4 Rylander R, Andersson K, Belin L, et al. Studies on humans exposed to airborne sewage sludge. Schweiz Med Wochenschr 1977;107:182-4.

5 Lundholm $M$, Rylander $R$. Work related symptoms among sewage workers. $\mathrm{Br} \mathcal{F}$ Ind Med 1983;40:325-9.

6 Rappaport SM, Richard MG, Hollstein MC, Talcott RE. Mutagenic activity in organic wastewater concentrates. Environmental Science and Technology 1979;13:957-61.

7 Elia VJ, Clark CS, Majeti VA et al. Hazardous chemical exposure at a municipal wastewater treatment plant. Environ Res 1983;32:360-71.

8 Scarlett-Kranz JM, Babish JG, Strickland D, Goodrich RM, Lisk DJ. Urinary mutagens in municipal sewage workers and water treatment workers. Am f Epidemiol 1986;124:884-93.

9 Olin RG, Ahlbom A, Lindberg-Navier I, Norell SE, Spännare B. Occupational factors associated with astrocytomas: a case-control study. Am f Ind Med 1987;11:615-25.

10 Lafleur J, Vena JE. Retrospective cohort mortality study of cancer among sewage plant workers. Am f Ind Med 1991;19:75-86.

11 Breslow NE, Day NE. Statistical methods in cancer research. Vol II. The design and analysis of cohort studies. Lyon: International Agency for Research on Cancer, 1987. (IARC sci pub No 82).

12 Nomura A, Stemmermann GN, Chyou P-H, Kato I, PerezPerez GI, Blaser MJ. Helicobacter pylori infection and gastric carcinoma among Japanese Americans in Hawaii. N Engl $\mathcal{F}$ Med 1991;325:1132-6.

13 Parsonnet J, Friedman GD, Vandersteen DP, et al. Helicobacter pylori infection and the risk of gastic carcinoma. $N$ Engl F Med 1991;325:1127-31.

Accepted 5 October 1992 